\title{
Risk of developing severe sepsis after acute kidney injury: a population-based cohort study
}

\author{
Tai-Shuan Lai ${ }^{1,2}$, Cheng-Yi Wang ${ }^{3}$, Sung-Ching Pan ${ }^{4}$, Tao-Min Huang ${ }^{5}$, Meng-Chun Lin ${ }^{4}$, Chun-Fu Lai ${ }^{4}$, \\ Che-Hsiung $\mathrm{Wu}^{6}$, Vin-Cent Wu $\mathrm{u}^{4^{*}}$, Kuo-Liong Chien ${ }^{2^{*}}$ and on behalf of the National Taiwan University Hospital \\ Study Group on Acute Renal Failure (NSARF)
}

\begin{abstract}
Introduction: Sepsis has been a factor of acute kidney injury (AKI); however, little is known about dialysis-requiring AKI and the risk of severe sepsis after survival to discharge.

Methods: We conducted a population-based cohort study based on the Taiwan National Health Insurance Research Database from 1999 to 2009. We identified patients with AKI requiring dialysis during hospitalization and survived for at least 90 days after discharge, and matched them with those without AKI according to age, sex, and concurrent diabetes. The primary outcome was severe sepsis, defined as sepsis with a diagnosis of acute organ dysfunction. Individuals who recovered enough to survive without acute dialysis were further analyzed.

Results: We identified 2983 individuals (mean age, 62 years; median follow-up, 3.96 years) with dialysis-requiring AKI and 11,932 matched controls. The incidence rate of severe sepsis was 6.84 and 2.32 per 100 person-years among individuals with dialysis-requiring AKI and without AKI in the index hospitalization, respectively. Dialysisrequiring AKI patients had a higher risk of developing de novo severe sepsis than the non-AKI group. In subgroup analysis, even individuals with recovery from dialysis-requiring AKI were at high risk of developing severe sepsis.
\end{abstract}

Conclusions: AKI is an independent risk factor for severe sepsis. Even patients who recovered from AKI had a high risk of long-term severe sepsis.

\section{Introduction}

Sepsis can cause multiorgan dysfunction, especially of the heart, lung and kidney, causing high morbidity and mortality in hospitalized patients [1]. Severe sepsis, defined as sepsis with organ dysfunction, hypoperfusion or hypotension, occurs in about $11 \%$ to $27 \%$ of ICU patients, with a mortality rate of about $18 \%$ to $55 \%$ [2-4]. The incidence and severity of sepsis has increased in recent years and has contributed substantially to the disease burden over time [5].

AKI is a common complication, especially in critically ill patients, occurring in $8 \%$ of hospitalized patients and in approximately $50 \%$ of ICU patients [6]. Sepsis is the most common cause of AKI. Severe sepsis, including septic shock, accounts for almost half of all cases of AKI in the

\footnotetext{
* Correspondence: d99849016@ntu.edu.tw; klchien@ntu.edu.tw

${ }^{2}$ Institute of Epidemiology and Preventive Medicine, National Taiwan

University, 17 Xu-Zhou Road, Taipei 10055, Taiwan

${ }^{4}$ Department of Internal Medicine, National Taiwan University Hospital,

7 Chung-Shan S Rd, Taipei 100, Taiwan

Full list of author information is available at the end of the article
}

ICU $[7,8]$. In humans, extrarenal organ dysfunction frequently coexists with AKI, potentiating the already high rates of AKI-associated morbidity and mortality. Unique systemic inflammatory patterns have also been observed for different mechanisms of AKI [9], as the deleterious interaction arises, at least in part, from systemic inflammatory changes, activation of proapoptotic pathways, increases in leukocyte trafficking and dysregulated channel expression [10]. These in turn can lead to long-term sepsis. The aim of this study was to evaluate the incidence of severe sepsis after hospitalization in patients with or without AKI requiring dialysis in a large-scale, populationbased administration database in Taiwan. We hypothesized that AKI might be an independent risk factor for developing severe sepsis, regardless of recovery of renal function recovery.

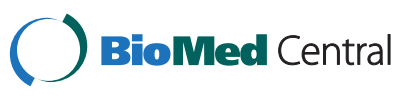

(C) 2013 Lai et al.; licensee BioMed Central Ltd. This is an open access article distributed under the terms of the Creative Commons Attribution License (http://creativecommons.org/licenses/by/2.0), which permits unrestricted use, distribution, and reproduction in any medium, provided the original work is properly cited. 


\section{Materials and methods Data sources}

The National Health Insurance (NHI) is a nationwide, compulsory, comprehensive health system in Taiwan. Patients were drawn from the NHI Research Database (NHIRD), which was released for research purposes by the National Health Research Institutes, Taipei, Taiwan [11]. The NHIRD, one of the largest databases in the world, covers nearly all (99\%) inpatient and outpatient claims for its population of more than 22 million people, and it has been used extensively in previous studies $[5,12]$. The NHIRD provides encrypted patient identification numbers; age; sex; dates of hospital admission and discharge; medical institutions providing services; International Classification of Diseases, Ninth Edition, Clinical Modification (ICD-9-CM), codes of diagnoses and procedures; and outcome at hospital discharge (recovered, died or transferred out).

\section{Patient selection and definition}

The NHIRD consists of all the original claim data and registration files of one million individuals from 1999 to 2008. These one million individuals were randomly sampled from the 2000 Registry for Beneficiaries of the Taiwan NHI program and screening comorbidities by using the medical records for one year preceding admission. We linked to diagnostic codes through the hospitalization claims data to identify all episodes of AKI and the corresponding order codes used for the study subjects. We defined the first AKI hospitalization requiring dialysis as the index hospitalization for each individual. Participants who received dialysis during the index hospitalization were included. We excluded individuals who had a previous diagnosis of AKI, had received a kidney transplant or any form of dialysis preceding the index hospitalization AKI $(n=6,420)$, were hospitalized for more than 180 days with AKI $(n=36)$, died during the index hospitalization AKI $(n=718)$ or withdrew from the NHI within three months after discharge $(n=1,382)$. Each AKI patient was then matched with four hospitalized patients without AKI according to age, sex and history of diabetes (Figure 1). Informed consent was originally obtained by the NHRI, and, since patients were anonymous in the present study, informed consent was not required. Also, since the identification numbers of all individuals in the NHRID were encrypted to protect the privacy of the individuals, this study was exempt from a full ethical review by the institutional ethics review board of National Taiwan University Hospital (reference no. 201212021RINC).

\section{Ascertainment of study outcome}

Patients were tracked for outcomes beginning 90 days after discharge and ending at 31 December 2009. The main study outcome was severe sepsis. The method of identification of patients with severe sepsis was similar to that used by Angus et al. [3], who selected all acute care hospitalizations with ICD-9-CM codes for both a bacterial or fungal infection process and a diagnosis of

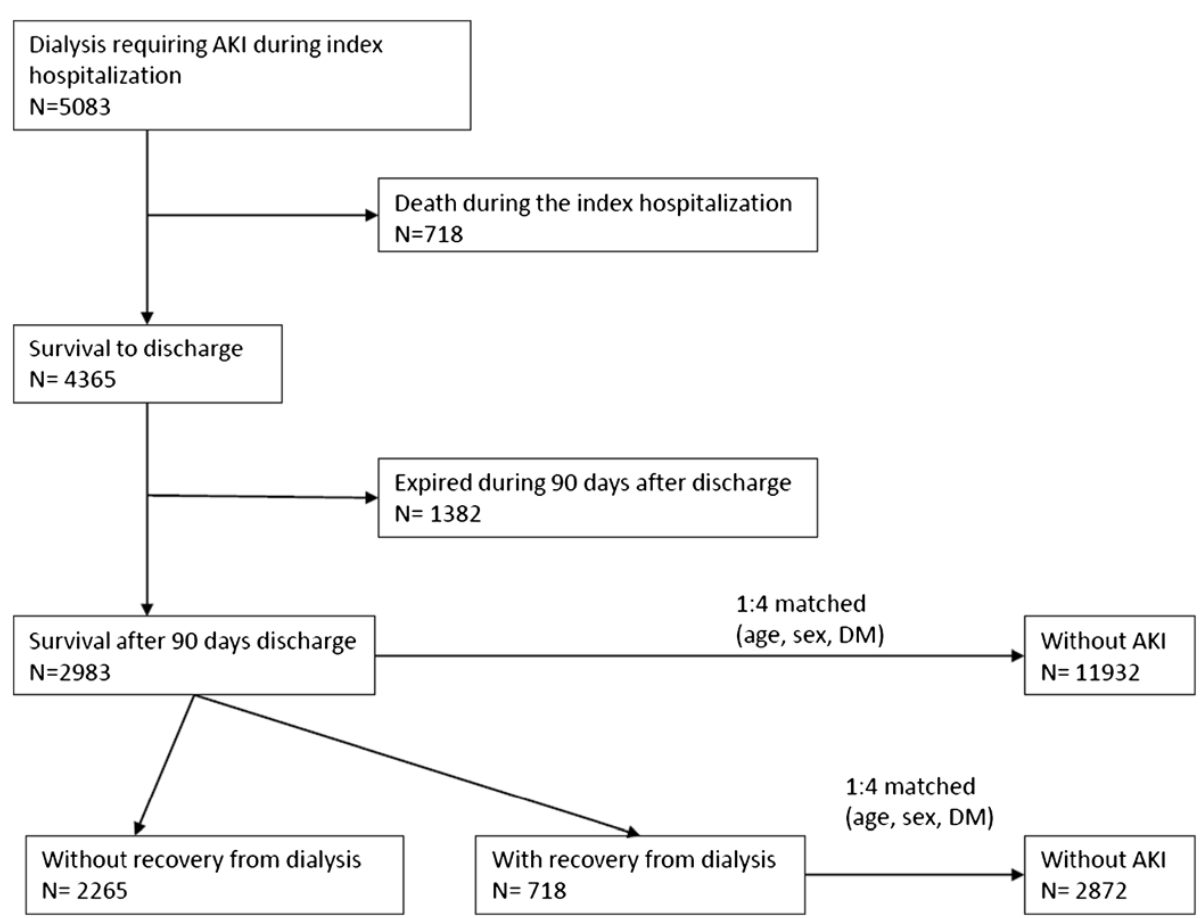

Figure 1 Flow diaphragm of the study population. AKl: acute kidney injury. 
acute organ dysfunction. We used codes for acute organ dysfunction as modified by Shen et al. [5] (listed in Table 1). Sepsis was defined according to the American College of Chest Physicians/Society of Critical Care

Table 1 Acute organ dysfunction code for severe sepsis

\begin{tabular}{|c|c|c|}
\hline $\begin{array}{l}\text { Organ } \\
\text { dysfunction }\end{array}$ & Codes & Code description \\
\hline \multirow[t]{7}{*}{ Cardiovascular } & 458.0 & Hypotension, postural \\
\hline & 458.8 & $\begin{array}{l}\text { Hypotension, specified type, not } \\
\text { elsewhere classified }\end{array}$ \\
\hline & 458.9 & Hypotension, arterial, constitutional \\
\hline & 785.5 & Shock \\
\hline & 785.51 & Shock, cardiogenic \\
\hline & 785.59 & Shock, circulatory or septic \\
\hline & 796.3 & Hypotension, transient \\
\hline \multirow[t]{8}{*}{ Respiratory } & 518.81 & Acute respiratory failure \\
\hline & 518.82 & $\begin{array}{l}\text { Acute respiratory distress syndrome } \\
\text { (ARDS) }\end{array}$ \\
\hline & 518.85 & ARDS after shock or trauma \\
\hline & 786.09 & Respiratory insufficiency \\
\hline & 799.1 & Respiratory arrest \\
\hline & $\begin{array}{l}96.7 \\
(96.71,96.72)\end{array}$ & Ventilator management \\
\hline & 96.04 & $\begin{array}{l}\text { Endotracheal intubation (emergency } \\
\text { procedure) }\end{array}$ \\
\hline & 93.9 & Continuous positive airway pressure \\
\hline \multirow[t]{4}{*}{ Renal } & $580 . x$ & Acute glomerulonephritis \\
\hline & 584.x & Acute renal failure \\
\hline & 586 & Renal shutdown, renal failure unspecified \\
\hline & 39.95 & Hemodialysis \\
\hline \multirow[t]{4}{*}{ Hepatic } & 570 & Acute hepatic failure or necrosis \\
\hline & 572.2 & Hepatic encephalopathy \\
\hline & 573.3 & $\begin{array}{l}\text { Hepatitis (septic and not elsewhere } \\
\text { classified) }\end{array}$ \\
\hline & 573.4 & Hepatic infarction \\
\hline \multirow[t]{6}{*}{ Neurologic } & 293 & Transient organic psychosis \\
\hline & 348.1 & Anoxic brain injury \\
\hline & 348.3 & Encephalopathy, acute \\
\hline & 780.01 & Coma \\
\hline & 780.09 & Altered consciousness, unspecified \\
\hline & 89.14 & Electroencephalography \\
\hline \multirow[t]{5}{*}{ Hematologic } & 286.2 & Disseminated intravascular coagulation \\
\hline & 286.6 & Purpura fulminans \\
\hline & 286.9 & Coagulopathy \\
\hline & $287.3-5$ & $\begin{array}{l}\text { Thrombocytopenia, primary, secondary or } \\
\text { unspecified }\end{array}$ \\
\hline & 790.92 & Abnormal coagulation profile \\
\hline Metabolic & 276.2 & Acidosis, metabolic or lactic \\
\hline
\end{tabular}

Medicine (ACCP/SCCM) as systemic inflammatory syndrome in response to infection, which, when associated with acute organ dysfunction, is said to be severe [2]. For patients with more than one hospital admission for severe sepsis during the study period, the first episode of sepsis was included.

\section{Statistical analyses}

For individuals with and those without AKI and dialysis, continuous variables were compared using an unpaired $t$-test and are expressed as mean values with SD. Categorical variables were compared using a $\chi^{2}$ test and expressed as a percentage. The person-years were calculated from enrollment to outcome, death or the end of the study period. Incidence rates of severe sepsis were calculated for participants with and those without AKI and dialysis, respectively.

Each acute dialysis patient was matched with four non-AKI individuals according to age, sex and history of diabetes. Crude hazard ratios (HRs) with 95\% confidence intervals (CIs) were derived from Cox proportional hazards models, and matched individuals without AKI or dialysis constituted the reference group. A KaplanMeier curve was generated from unadjusted hazards models. We further adjusted for age, sex, comorbidities, procedure codes and a propensity score in the multivariate models. Subsequent advanced chronic kidney disease (CKD) after discharge was evaluated for its effect on long-term severe sepsis using a time-dependent Cox analysis. The adjusted HR values for severe sepsis were further stratified according to comorbidities.

We used a propensity score approach to account for baseline differences between the AKI groups. Multiple logistic regressions were used to generate propensity scores, which were the predicted probabilities of AKI requiring dialysis. Covariates that were statistically significant in the unadjusted analysis were fit into the model, including age; ICU admission during the index hospitalization; admission frequency; use of mechanical ventilation; comorbidities before admission, such as diabetes mellitus, tumor with metastasis, CKD, chronic obstructive pulmonary disease and congestive heart failure; and comorbidities during index hospitalization, such as those of renal, metabolic, pulmonary or cardiovascular origin. We incorporated this score into a Cox proportional hazards model as a covariate.

In subgroup analysis, each acute dialysis patient with recovery from dialysis was matched with patients without AKI according to age, sex and history of diabetes. A two-sided $P$ value less than 0.05 was considered statistically significant. All data were analyzed using $\mathrm{R}$ statistical software (version 2.14.1; Free Software Foundation, Inc, Boston, MA, USA). 


\section{Results}

A total of 2,983 individuals with AKI requiring dialysis who survived at least 90 days after hospital discharge were identified, and 11,932 individuals without dialysis or AKI were matched. Baseline characteristics between individuals with or without AKI acute dialysis are listed in Table 2. The acute dialysis group had more macrovascular and microvascular disease than the non-AKI group.
Among the 2,983 individuals with AKI requiring dialysis, 2,265 patients remained on dialysis and 718 recovered from AKI requiring dialysis after 90 days postdischarge.

The mean age of the enrolled participants was $62 \pm$ 14.8 years, and $50.3 \%$ were men. Compared with nonAKI participants, those with acute dialysis had a higher mean Charlson Comorbidity Index score $(3.7 \pm 2.2$ vs. $1.7 \pm 1.7)$ and more comorbidities before the index

Table 2 Baseline characteristics of study population stratified by acute kidney injury ${ }^{a}$

\begin{tabular}{|c|c|c|c|}
\hline Patient characteristics & $\begin{array}{l}\text { Non-AKI group } \\
(N=11,932)\end{array}$ & $\begin{array}{l}\text { AKI group } \\
(N=2,983)\end{array}$ & $P$-value \\
\hline Mean age (years) & $62.03 \pm 14.84$ & $62.03 \pm 14.84$ & 0.995 \\
\hline Males (\%) & $6,004(50.3 \%)$ & $1,501(50.3 \%)$ & 0.999 \\
\hline Admission frequency & $0.57 \pm 1.61$ & $2.28 \pm 2.75$ & $<0.001$ \\
\hline Charlson Comorbidity Index score & $1.67 \pm 1.72$ & $3.66 \pm 2.17$ & $<0.001$ \\
\hline \multicolumn{4}{|l|}{ Comorbidities (before admission) } \\
\hline Myocardial infarction & $122(1 \%)$ & $89(3 \%)$ & $<0.001$ \\
\hline Congestive heart failure & $426(3.6 \%)$ & $549(18.4 \%)$ & $<0.001$ \\
\hline Peripheral vascular disease & $161(1.3 \%)$ & $102(3.4 \%)$ & $<0.001$ \\
\hline Cerebrovascular disease & $1,117(9.4 \%)$ & $477(16 \%)$ & $<0.001$ \\
\hline Dementia & $204(1.7 \%)$ & $87(2.9 \%)$ & $<0.001$ \\
\hline COPD & $1,854(15.5 \%)$ & $497(16.7 \%)$ & 0.137 \\
\hline Rheumatologic disease & $158(1.3 \%)$ & $55(1.8 \%)$ & 0.038 \\
\hline Peptic ulcer & $2,182(18.3 \%)$ & $786(26.3 \%)$ & $<0.001$ \\
\hline Moderate or severe liver disease & $1,288(10.8 \%)$ & $285(9.6 \%)$ & 0.049 \\
\hline Diabetes mellitus & $5,973(50.1 \%)$ & $1,494(50.1 \%)$ & 0.984 \\
\hline Hemiplegia & $147(1.2 \%)$ & $68(2.3 \%)$ & $<0.001$ \\
\hline Chronic kidney disease & $845(7.1 \%)$ & $2,248(75.4 \%)$ & $<0.001$ \\
\hline Solid tumor & $657(5.5 \%)$ & $193(6.5 \%)$ & 0.047 \\
\hline Tumor with metastasis & $198(1.7 \%)$ & $40(1.3 \%)$ & 0.252 \\
\hline \multicolumn{4}{|l|}{ Comorbidities (during index hospitalization) } \\
\hline Cardiovascular & $89(0.7 \%)$ & $84(2.8 \%)$ & $<0.001$ \\
\hline Respiratory & $100(0.8 \%)$ & $286(9.6 \%)$ & $<0.001$ \\
\hline Hepatic & $96(0.8 \%)$ & $36(1.2 \%)$ & 0.048 \\
\hline Neurologic & $18(0.2 \%)$ & $48(1.6 \%)$ & $<0.001$ \\
\hline Hematologic & $56(0.5 \%)$ & $29(1 \%)$ & 0.002 \\
\hline Metabolic & $5(0 \%)$ & $98(3.3 \%)$ & $<0.001$ \\
\hline \multicolumn{4}{|l|}{ Operative categories } \\
\hline Cardiothoracic & $78(0.7 \%)$ & $51(1.7 \%)$ & $<0.001$ \\
\hline Upper Gl & $72(0.6 \%)$ & $12(0.4 \%)$ & 0.219 \\
\hline Lower Gl & $128(1.1 \%)$ & $20(0.7 \%)$ & 0.049 \\
\hline Hepatobiliary & $197(1.7 \%)$ & $13(0.4 \%)$ & $<0.001$ \\
\hline ICU admission during index hospitalization & $898(7.5 \%)$ & $948(31.8 \%)$ & $<0.001$ \\
\hline Mechanical ventilation & $376(3.2 \%)$ & $527(17.7 \%)$ & $<0.001$ \\
\hline Mortality & $2603(21.8 \%)$ & $1,386(46.5 \%)$ & $<0.001$ \\
\hline Severe sepsis & 1,250 (10.5\%) & 675 (22.6\%) & $<0.001$ \\
\hline
\end{tabular}

${ }^{a} A K I$ : acute kidney injury; COPD: chronic obstructive pulmonary disease; GI: gastrointestinal. 
hospitalization, including cardiovascular, peripheral vascular and cerebrovascular disease. Participants with acute dialysis had a much higher prevalence of underlying CKD (75.4\% vs. $7.1 \%)$ than did non-AKI patients. During their hospitalization, patients with acute dialysis were more likely to receive mechanical ventilation (17.7 vs. $3.2 \% ; P<0.001)$, had higher long-term mortality ( 46.5 vs. $21.8 \% ; P<0.001)$ and developed severe sepsis more often (22.6 vs. $10.5 \%)$ than did patients without AKI. The respective rates for severe sepsis were $22.6 \%$ and $10.5 \%$.

After a median follow-up period of 3.96 years, the incidence rates of severe sepsis were 6.84 and 2.32 per 100 person-years among individuals with and those without AKI and dialysis in the index hospitalization, respectively, resulting in a HR of 2.87 (95\% CI, 2.62 to 3.16 ; $P<$ $0.001)$. The propensity score-adjusted HR was 1.83 (95\% CI, 1.57 to $2.13 ; P<0.001)$. After multivariable adjustment, patients with AKI requiring dialysis were at a higher risk than patients in the non-AKI group for developing de novo severe sepsis (HR: 1.99 (95\% CI: 1.71 to 2.31 ); $P<0.001$ ) (Table 3 ). This model had good validity with a C-index concordance statistic of 0.79 and an adjusted generalized $R^{2}$ of 0.12 . The Cox proportional hazards model is plotted in Figure 2. In time-dependent analysis, the AKI group requiring dialysis had a higher risk of developing severe sepsis than the non-AKI group in the first year and decreased gradually with time (oneyear HR: 3.44 (95\% CI: 2.59 to 4.56 ), two-year HR: 2.26 (95\% CI: 1.83 to 2.78 ] and three-year HR: 2.05 (95\% CI: 1.70 to 2.48), respectively) (Table 4). For participants

Table 3 Crude and adjusted hazard ratios of severe sepsis for acute kidney injury status ${ }^{a}$

\begin{tabular}{|c|c|c|c|c|}
\hline \multirow{2}{*}{$\begin{array}{l}\text { Analysis } \\
\text { model }\end{array}$} & \multicolumn{2}{|c|}{ AKI vs. non-AKI } & \multicolumn{2}{|c|}{ Recovery vs. non-AKI } \\
\hline & HR (95\% Cl) & $P$-value & HR (95\% Cl) & $P$-value \\
\hline Univariate & 2.87 (2.62 to 3.16 ) & $<0.001$ & 2.80 (2.34 to 3.34$)$ & $<0.001$ \\
\hline \multicolumn{5}{|l|}{ Multivariate } \\
\hline Model 1 & $1.83(1.57-2.13)$ & $<0.001$ & 1.48 (1.06 to 2.06$)$ & 0.02 \\
\hline Model 2 & 1.99 (1.71 to 2.31$)$ & $<0.001$ & & \\
\hline Model 3 & & & 1.58 (1.15 to 2.16$)$ & $<0.001$ \\
\hline Model 4 & 1.95 (1.67 to 2.28 ) & $<0.001$ & & \\
\hline
\end{tabular}

${ }^{a}$ AKI: acute kidney injury; HR: hazard ratio. Model 1: Adjusted for age, sex and propensity score.

Model 2: Adjusted for age, sex, propensity score, ICU admission, admission frequency, Charlson Comorbidity Index score, comorbidities before index hospitalization (cerebrovascular disease, dementia, chronic obstructive pulmonary disease, hemiplegia and metastatic tumor) and respiratory and hematologic comorbidities during index hospitalization. Model 3: Adjusted for age, sex, propensity score, admission frequency, Charlson Comorbidity Index score, comorbidities before index hospitalization (peptic ulcer and diabetes mellitus) and cardiovascular and respiratory comorbidities during index hospitalization. Model 4: Model 2 adjusted with subsequent advanced CKD after hospital discharge as a time-varying covariate. Advanced CKD is defined as patients with serum creatinine levels above $6 \mathrm{mg} / \mathrm{dl}$ and a concomitant erythropoiesis-stimulating agent prescription.

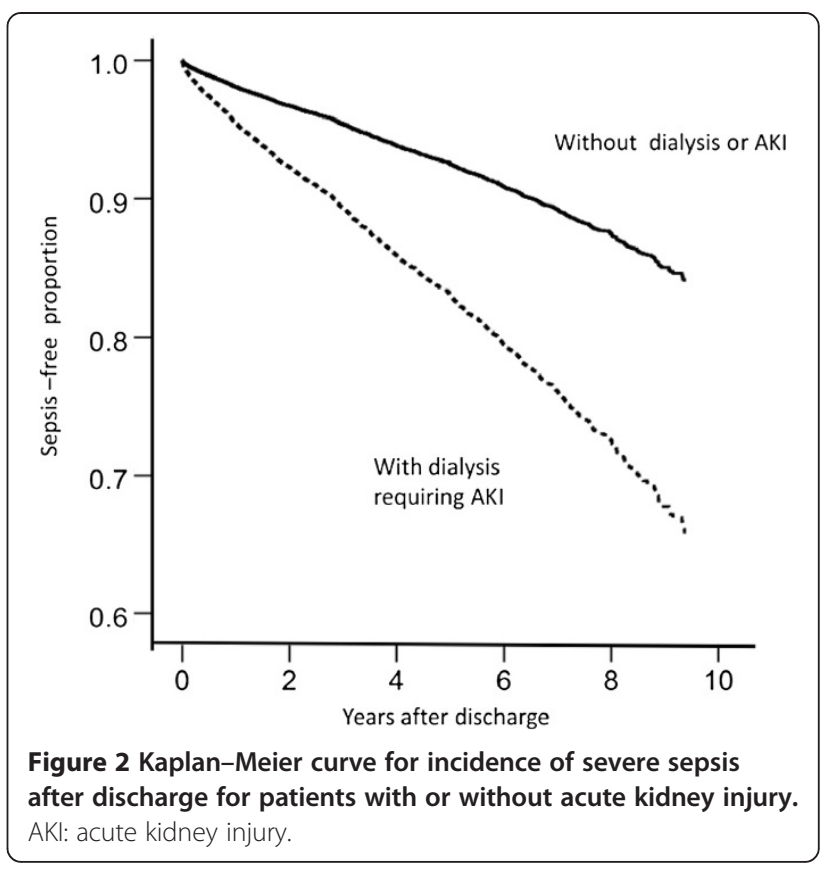

with and those without recovery from AKI and dialysis, the tendency for the development of severe sepsis did a $95 \% \mathrm{CI}$ of 0.9 to 1.34 ( $P=0.457)$. The increased risk of developing long-term severe sepsis following hospitalization complicated by AKI requiring dialysis was consistent across patient subgroups, including cerebrovascular disease, chronic obstructive pulmonary disease, hemiplegia and diabetes mellitus (Figure 3).

In a subgroup analysis, we compared individuals who recovered from AKI requiring dialysis with non-AKI individuals. The crude HR and propensity score-adjusted HRs were 2.80 (95\% CI: 2.34 to 3.34; $P<0.001$ ) and 1.48 (95\% CI: 1.06 to $2.06 ; P=0.02$ ), respectively. The recovery group was at a higher risk for severe sepsis than the non-AKI group after multivariable adjustment for the covariates listed in Table 3 (HR: 1.58 (95\% CI: 1.15 to 2.16); $P<0.001$ ). This model also showed good validity, with a C-index concordance statistic of 0.78 and an adjusted generalized $R^{2}$ of 0.15 .

\section{Discussion}

AKI is an independent predictor of increasing mortality in critically ill patients $[13,14]$. Our study demonstrates that AKI requiring dialysis is associated with subsequent long-term severe sepsis. After a median follow-up of 3.96 years, the incidence of severe sepsis in the AKI group was about threefold the incidence of severe sepsis in the non-AKI group. In a subgroup analysis, we found that, even in individuals who recovered from AKI and dialysis, the incidence of severe sepsis was $58 \%$ higher than that in individuals without AKI, suggesting that 
Table 4 Time-dependent analysis of the risk of developing severe sepsis ${ }^{a}$

\begin{tabular}{|c|c|c|c|c|}
\hline \multirow[t]{2}{*}{ Model } & \multicolumn{2}{|l|}{ AKI vs. non-AKI } & \multicolumn{2}{|c|}{ Recovery vs. non-AKI } \\
\hline & $\mathrm{HR}(95 \% \mathrm{Cl})$ & $P$-value & HR $(95 \% \mathrm{CI})$ & $P$-value \\
\hline Model 5 & 3.44 (2.59 to 4.56$)$ & $<0.001$ & 1.61 (1.00 to 2.59$)$ & 0.049 \\
\hline
\end{tabular}

(one year of

follow-up)

Model $6 \quad 2.26(1.83$ to 2.78$)<0.001 \quad 1.61(1.09$ to 2.37$) \quad 0.017$

(two years of

follow-up)

Model $7 \quad 2.05(1.70$ to 2.48$)<0.001 \quad 1.68(1.18$ to 2.40$) \quad 0.004$

(three years of

follow-up)

${ }^{\mathrm{a} A K I}$ : acute kidney injury. (a) AKI group vs. non-AKI group. (b) Recovery group vs. non-AKI group). Model 5a: Adjusted for age, propensity score, admission frequency, comorbidities before index hospitalization (chronic kidney disease (CKD), cerebrovascular disease, dementia, chronic pulmonary disease and metastatic tumor), hematologic comorbidities during index hospitalization and advanced CKD. Advanced CKD is defined as patients with serum creatinine levels above $6 \mathrm{mg} / \mathrm{dl}$ and a concomitant erythropoiesis-stimulating agent prescription. Model 5b: Adjusted for age, propensity score, admission frequency, Charlson Comorbidity Index score, comorbidities before index hospitalization (cerebrovascular disease and peptic ulcer) and cardiovascular and respiratory comorbidities during index hospitalization. Model 6a: Adjusted for age, propensity score, Charlson Comorbidity Index score, admission frequency, comorbidities before index hospitalization (CKD, cerebrovascular disease, dementia and chronic obstructive pulmonary disease), metabolic and respiratory comorbidities during index hospitalization and advanced CKD. Model 6b: Adjusted for age, propensity score, admission frequency, Charlson Comorbidity Index score, comorbidities before index hospitalization (cerebrovascular disease and peptic ulcer) and cardiovascular, respiratory and hepatic comorbidities during index hospitalization. Model 7a: Adjusted for age, propensity score, Charlson Comorbidity Index score, admission frequency, comorbidities before index hospitalization (CKD, cerebrovascular disease, dementia and chronic obstructive pulmonary disease) and metabolic, hematologic and respiratory comorbidities during index hospitalization. Model 7b: Adjusted for age, propensity score, admission frequency, Charlson Comorbidity Index score, comorbidities before index hospitalization (cerebrovascular disease and peptic ulcer) and respiratory comorbidities during index hospitalization.

AKI per se is an independent risk factor for developing severe sepsis, regardless of recovery from AKI. The prevalence rate of severe sepsis was 2.32 per 100 personyears in the non-AKI group, which is consistent with the national estimate of 2.26 per 100 person-years in hospital patients in the United States [3].

The prevalence of sepsis after AKI has been underestimated, and, until now, only a few studies have emphasized the effect of AKI on the incidence of sepsis. For example, a cohort study of contrast-induced AKI illustrated that $45 \%$ of patients developed sepsis subsequently during hospitalization, but the long-term development of sepsis was not reported [15]. Another study retrospectively investigated the incidence of infection in critically ill patients with AKI and found that $80 \%$ of critical ill patients with AKI developed infections during hospitalization [16]. Of all episodes of infection, $46.2 \%$ occurred before, $40.3 \%$ during and $13.4 \%$ after discontinuation of renal replacement therapy. Single-center enrollment or a small sample size limits the robustness of studies, however, and the definition of sepsis is often overestimated. Recently, in a multicenter, observational study, Mehta et al. analyzed data from 618 critically ill patients in the ICU and examined the relationship between sepsis and AKI [17]. The mortality rate for patients with who developed sepsis after AKI was higher than that for sepsis-free patients ( $44 \%$ vs. $21 \% ; P<0.001)$ and similar to that for patients with sepsis preceding AKI ( $48 \%$ vs. $44 \%$; $P=0.41)$. By using comprehensive administrative data in Taiwan in our present study, we have provided strong evidence that AKI is an independent risk factor for developing severe sepsis, even when it occurs a long time after index hospitalization. Furthermore, although dialysis was an important predictor of sepsis after AKI diagnosis, our results show that patients who recovered from dialysis still had a higher risk of subsequent severe sepsis, suggesting that chronic dialysis itself is not the crucial source of post-AKI sepsis in these patients.

Patients in the ICU are dying as a result of AKI, but not simply from AKI. Experimental and small observational studies have shown that AKI negatively affects immunity and is associated with higher rates of infection [18]. AKI patients frequently develop a vicious cycle of immune dysfunction, sepsis and multiorgan failure. Indeed, severe sepsis is currently the major cause of AKI in the United States [19]. The host response to sepsis involves an inflammatory response which activates innate immunity. If this persists, the immune response will lead to a release of a multitude of proinflammatory products, which frequently cause organ dysfunction, including kidney failure [20-26]. Our finding that patients with AKI were fragile and were more likely to develop severe sepsis reinforces the fact that acute renal dysfunction plays a crucial immunomodulatory role in individuals in stressed states. Also, AKI has been proved to facilitate organ cross-talk in the heart, brain, lungs, liver and other organs via the relevant biological pathways $[27,28]$. Multiple end-stage organ injury after AKI may worsen the severity of infection in severe sepsis.

Though renal function has been thought to be reversible after AKI, recent studies have provided evidence that AKI increases the risk of CKD and end-stage renal disease requiring dialysis $[7,29,30]$. A systematic review and meta-analysis showed a higher risk of CKD and chronic dialysis in patients after AKI [31]. Clinical studies have also demonstrated that CKD attenuated immunity and subsequently increased the risk of pneumonia, influenza and associated sepsis [32]. Thus, increased risk of severe sepsis, even among the recovery group, with subsequent CKD and impaired immunity is reasonable. In our analysis, we further adjusted for advanced CKD status represented by erythropoietin use, and we found that the AKI group requiring dialysis was still at a higher risk for developing severe sepsis (HR: 1.95 (95\% CI: 1.67 


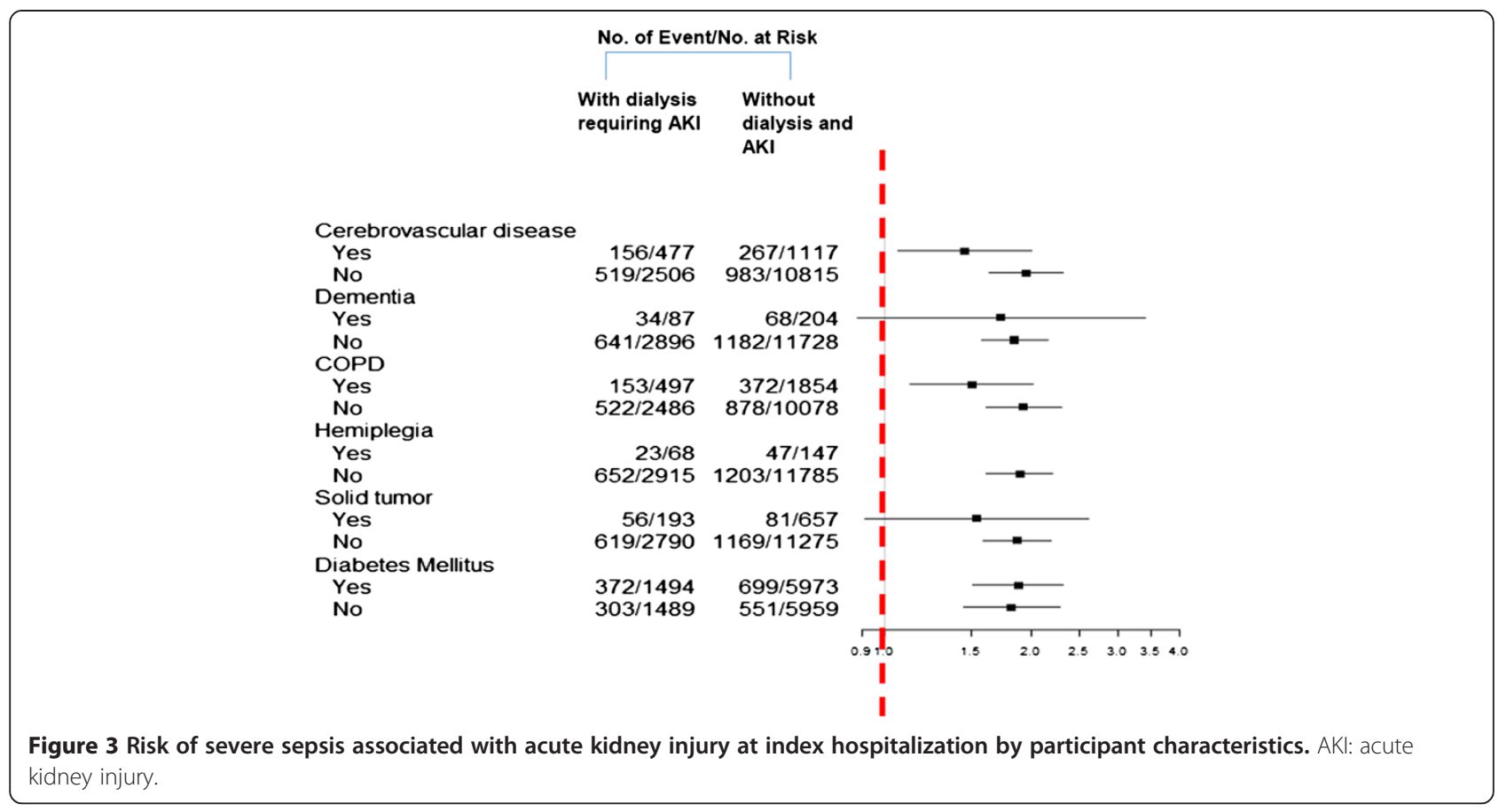

to 2.28); $P<0.001$ ), suggesting that patients with AKI are still at risk of developing severe sepsis after removal of the uremia effect.

Our study has several limitations. First, when using administrative databases, the diagnosis of AKI, severe sepsis and other comorbidities are totally based on ICD and procedure codes, so misclassification inevitably occurs. However, the misclassification is often nondifferential and the outcome difference is toward null. In a recent validation study, a diagnostic code for AKI plus a procedure code for dialysis had a sensitivity and specificity of more than $90 \%$ for capturing patients with AKI requiring dialysis [33]. Furthermore, the NHIB in Taiwan has randomly reviewed medical charts and audited all medical charges [34]; hence, the data quality is trustworthy. Second, individual information such as smoking, drinking and site of infection was not available through administrative data in the NHIRD. We were also unable to obtain detailed laboratory data, such as serum creatinine level or urinary protein excretion. There may be residual confounding factors caused by these unknown data. However, these factors have less effect on our outcome than the factors we adjusted for, and the robustness of the association between AKI and long-term severe sepsis is largely unaffected.

\section{Conclusions}

In this study, we have demonstrated that AKI is an independent risk factor for the development of long-term severe sepsis. The incidence of severe sepsis is threefold in patients with AKI requiring dialysis compared with non-
AKI patients. Our study further indicates that individuals who recovered from AKI and dialysis still had a higher risk than non-AKI patients of developing severe sepsis. Therefore, all AKI patients requiring dialysis, regardless they recover, should be considered at risk for severe sepsis and should be aggressively managed for infection prevention. Because of the high mortality in severe sepsis, awareness of the high risk of severe sepsis after AKI should be raised in outpatient clinical practice.

\section{Key messages}

- Sepsis has been a factor in patients with AKI; however, the long-term risk of severe sepsis in patients after AKI requiring dialysis may be overlooked.

- We have revealed that AKI is an independent risk factor for the development of long-term severe sepsis. The incidence of severe sepsis in the AKI group was about threefold the incidence of severe sepsis in the non-AKI group after a median of four years follow-up.

- We also revealed that even patients who recovered from AKI had a high risk of long-term severe sepsis. Therefore, we should view AKI as not only a selflimiting acute disease but also a long-lasting immunomodulatory disorder.

- We need to raise the awareness of the high risk of severe sepsis after AKI in outpatient clinical practice. All AKI patients requiring dialysis, regardless of whether they recover, should be aggressively managed for infection prevention. 


\section{Abbreviations}

ACCP/SCCM: American College of Chest Physicians/Society of Critical Care Medicine; AKI: Acute kidney injury; Cl: Confidence interval; CKD: Chronic kidney disease; COPD: Chronic obstructive pulmonary disease; DM: Diabetes mellitus; HR: Hazard ratio; ICD-9-CM: International Classification of Diseases, Ninth Edition, Clinical Modification; NHI: National Health Insurance; NHIRD: National Health Insurance Research Database.

\section{Competing interests}

The authors declare that they have no competing interests.

\section{Authors' contributions}

TSL conceived the study, participated in data collection, performed statistical analysis, interpreted the results and wrote the manuscript. CYW, SCP, TMH, CFL and VCW participated in data collection and manuscript revision. MCL and CHW participated in data collection. VCW and KLC conceived the study and participated in manuscript revision. All authors read and approved the final manuscript.

\section{Authors' information}

The National Taiwan University Hospital Study Group for Acute Renal Failure (NSARF) includes Wen-Je Ko, Vin-Cent Wu, Chun-Fu Lai, Tao-Min Huang, TaiShuan Lai, Yung-Ming Chen, Chih-Chung Shiao, Wei-Shun Yang, Wei-Jie Wang, Likwang Chen, Cheng-Yi Wang, Pei-Chen Wu, Pi-Ru Tsai, Yu-Chang Yeh, Fu-Chang Hu and Kwan-Dun Wu.

\section{Acknowledgements}

This study is based in part on data from the NHIRD provided by the Bureau of NHI, Department of Health, Taiwan, and managed by the NHRI. The interpretations and conclusions reported herein do not represent those of the Bureau of NHI, Department of Health or the NHRI. The authors thank all the participants in the National Taiwan University Hospital Study Group for Acute Renal Failure.

\section{Author details}

'Department of Internal Medicine, National Taiwan University Hospital, Bei-Hu Branch, 87 Neijiang St, Taipei 108, Taiwan. ${ }^{2}$ Institute of Epidemiology and Preventive Medicine, College of Public Health, National Taiwan University, 17 Xu-Zhou Rd, Taipei 100, Taiwan. ${ }^{3}$ Department of Internal Medicine, Cardinal Tien Hospital, 362 Zhongzheng Rd, New Taipei City 231, Taiwan. ${ }^{4}$ Department of Internal Medicine, National Taiwan University Hospital, 7 Chung-Shan S Rd, Taipei 100, Taiwan. ${ }^{5}$ Department of Internal Medicine, National Taiwan University Hospital, Yun-Lin Branch, 579 Sec 2, Yunlin Rd, Douliou City, Yunlin County 640, Taiwan. ${ }^{6}$ Department of Internal Medicine, Buddhist Tzu Chi General Hospital, 289 Jianguo Rd, New Taipei City 231, Taiwan.

Received: 17 April 2013 Accepted: 11 September 2013

Published: 11 October 2013

\section{References}

1. Sierra R: Sepsis and the kidney: Will the leaves fall off the tree? Crit Care Med 2012, 40:2247-2248

2. Bone RC, Balk RA, Cerra FB, Dellinger RP, Fein AM, Knaus WA, Schein RM, Sibbald WJ, The ACCP/SCCM Consensus Conference Committee: Definitions for sepsis and organ failure and guidelines for the use of innovative therapies in sepsis. Chest 1992, 101:1644-1655.

3. Angus DC, Linde-Zwirble WT, Lidicker J, Clermont G, Carcillo J, Pinsky MR: Epidemiology of severe sepsis in the United States: analysis of incidence, outcome, and associated costs of care. Crit Care Med 2001, 29:1303-1310.

4. Brun-Buisson C: The epidemiology of the systemic inflammatory response. Intensive Care Med 2000, 26:S64-S74

5. Shen HN, Lu CL, Yang HH: Epidemiologic trend of severe sepsis in Taiwan from 1997 through 2006. Chest 2010, 138:298-304.

6. Hoste EA, Clermont G, Kersten A, Venkataraman R, Angus DC, De Bacquer D, Kellum JA: RIFLE criteria for acute kidney injury are associated with hospital mortality in critically ill patients: a cohort analysis. Crit Care 2006, 10:R73.

7. Uchino S, Kellum JA, Bellomo R, Doig GS, Morimatsu H, Morgera S, Schetz M, Tan I, Bouman C, Macedo E, Gibney N, Tolwani A, Ronco C, Beginning and Ending Supportive Therapy for the Kidney (BEST Kidney) Investigators:
Acute renal failure in critically ill patients: a multinational, multicenter study. JAMA 2005, 294:813-818.

8. Bagshaw SM, Lapinsky S, Dial S, Arabi Y, Dodek P, Wood G, Ellis P, Guzman J, Marshall J, Parrillo JE, Skrobik Y, Kumar A, Cooperative Antimicrobial Therapy of Septic Shock (CATSS) Database Research Group: Acute kidney injury in septic shock: clinical outcomes and impact of duration of hypotension prior to initiation of antimicrobial therapy. Intensive Care Med 2009, 35:871-881.

9. Hoke TS, Douglas IS, Klein CL, He Z, Fang W, Thurman JM, Tao Y, Dursun B, Voelkel NF, Edelstein CL, Faubel S: Acute renal failure after bilateral nephrectomy is associated with cytokine-mediated pulmonary injury. J Am Soc Nephrol 2007, 18:155-164.

10. Grams ME, Rabb H: The distant organ effects of acute kidney injury. Kidney Int 2012, 81:942-948.

11. Kim WY, Huh JW, Lim CM, Koh Y, Hong SB: Analysis of progression in risk, injury, failure, loss, and end-stage renal disease classification on outcome in patients with severe sepsis and septic shock. J Crit Care 2012, 27:104. e1-104.e7.

12. Wu CY, Chen YJ, Ho HJ, Hsu YC, Kuo KN, Wu MS, Lin JT: Association between nucleoside analogues and risk of hepatitis $B$ virus-related hepatocellular carcinoma recurrence following liver resection. JAMA 2012, 308:1906-1914.

13. Brivet $F G$, Kleinknecht $D J$, Loirat $P$, Landais $P J$ : Acute renal failure in intensive care units-causes, outcome, and prognostic factors of hospital mortality: a prospective, multicenter study. Crit Care Med 1996, 24:192-198.

14. Chertow GM, Burdick E, Honour M, Bonventre JV, Bates DW: Acute kidney injury, mortality, length of stay, and costs in hospitalized patients. J Am Soc Nephrol 2005, 16:3365-3370.

15. Levy EM, Viscoli CM, Horwitz RI: The effect of acute renal failure on mortality: a cohort analysis. JAMA 1996, 275:1489-1494.

16. Reynvoet E, Vandijck DM, Blot SI, Dhondt AW, De Waele JJ, Claus S, Buyle FM, Vanholder RC, Hoste EA: Epidemiology of infection in critically ill patients with acute renal failure. Crit Care Med 2009, 37:2203-2209.

17. Mehta RL, Bouchard J, Soroko SB, Ikizler TA, Paganini EP, Chertow GM Himmelfarb J, Program to Improve Care in Acute Renal Disease (PICARD) Study Group: Sepsis as a cause and consequence of acute kidney injury: Program to Improve Care in Acute Renal Disease. Intensive Care Med 2011, 37:241-248.

18. Singbartl K, Kellum JA: AKI in the ICU: definition, epidemiology, risk stratification, and outcomes. Kidney Int 2012, 81:819-825.

19. United States Renal Data System: USRDS 2010 Annual Data Report: Volumes 1 and 2. Bethesda, MD: National Institutes of Health, National Institute of Diabetes and Digestive and Kidney Diseases; 2010. Available at http://www. usrds.org/atlas10.aspx (accessed 16 October 2013).

20. Basile DP: The endothelial cell in ischemic acute kidney injury: implications for acute and chronic function. Kidney Int 2007, 72:151-156.

21. Kelly KJ: Distant effects of experimental renal ischemia/reperfusion injury. J Am Soc Nephrol 2003, 14:1549-1558.

22. Bellomo R, Wan L, Langenberg C, May C: Septic acute kidney injury: new concepts. Nephron Exp Nephrol 2008, 109:e95-e100.

23. Himmelfarb J, McMonagle E, Freedman S, Klenzak J, McMenamin E, Le P, Pupim LB, Ikizler TA, The PICARD Group: Oxidative stress is increased in critically ill patients with acute renal failure. J Am Soc Nephrol 2004, 15:2449-2456.

24. Havasi A, Borkan SC: Apoptosis and acute kidney injury. Kidney Int 2011 , 80:29-40.

25. Okusa MD: The inflammatory cascade in acute ischemic renal failure. Nephron 2002, 90:133-138.

26. Gonçalves GM, Zamboni DS, Câmara NO: The role of innate immunity in septic acute kidney injuries. Shock 2010, 34:22-26.

27. Li X, Hassoun HT, Santora R, Rabb H: Organ crosstalk: the role of the kidney. Curr Opin Crit Care 2009, 15:481-487.

28. White $L E$, Hassoun $H T$ : Inflammatory mechanisms of organ crosstalk during ischemic acute kidney injury. Int J Nephrol 2012, 2012:505197.

29. Ishani A, Xue JL, Himmelfarb J, Eggers PW, Kimmel PL, Molitoris BA, Collins AJ: Acute kidney injury increases risk of ESRD among elderly. J Am SoC Nephrol 2009, 20:223-228.

30. Wald R, Quinn RR, Luo J, Li P, Scales DC, Mamdani MM, Ray JG, University of Toronto Acute Kidney Injury Research Group: Chronic dialysis and death among survivors of acute kidney injury requiring dialysis. JAMA 2009, 302:1179-1185. 
31. Coca SG, Singanamala S, Parikh CR: Chronic kidney disease after acute kidney injury: a systematic review and meta-analysis. Kidney Int 2012, 81:442-448.

32. Honoré PM, Jacobs R, Boer W, Joannes-Boyau O: Sepsis and AKI: more complex than just a simple question of chicken and egg. Intensive Care Med 2011, 37:186-189.

33. Waikar SS, Wald R, Chertow GM, Curhan GC, Winkelmayer WC, Liangos O, Sosa MA, Jaber BL: Validity of International Classification of Diseases, Ninth Revision, Clinical Modification codes for acute renal failure. J Am Soc Nephrol 2006, 17:1688-1694.

34. Chen YC, Su YC, Lee CC, Huang YS, Hwang SJ: Chronic kidney disease itself is a causal risk factor for stroke beyond traditional cardiovascular risk factors: a nationwide cohort study in Taiwan. PLoS One 2012, 7: e36332.

doi:10.1186/cc13054

Cite this article as: Lai et al:: Risk of developing severe sepsis after acute kidney injury: a population-based cohort study. Critical Care 2013 17:R231.

\section{Submit your next manuscript to BioMed Central and take full advantage of:}

- Convenient online submission

- Thorough peer review

- No space constraints or color figure charges

- Immediate publication on acceptance

- Inclusion in PubMed, CAS, Scopus and Google Scholar

- Research which is freely available for redistribution 